\title{
Hanseníase Lepromatosa: Relato de um Caso do Tipo Histióide-Símile
}

\author{
Ana Carolina Serra Rodrigues', Danielle Carvalho Quintella², Felipe Tavares Rodrigues ${ }^{3}$, Mercedes Prates Pockstaller ${ }^{4}$, José Augusto \\ da Costa Nery ${ }^{4}$ \\ 'Pós-Graduanda em Dermatologia do Instituto de Dermatologia Professor Rubem David Azulay. Hospital Geral da Santa Casa de \\ Misericórdia do Rio de Janeiro, Brasil \\ ${ }^{2}$ Patologista do Instituto de Dermatologia Professor Rubem David Azulay. Hospital Geral da Santa Casa de Misericórdia do Rio de \\ Janeiro, Brasil \\ ${ }^{3}$ Estudante de Medicina - Escola de Medicina e Cirurgia do Rio de Janeiro - Universidade Federal do Estado do Rio de Janeiro - \\ UNIRIO, Brasil. \\ ${ }_{4}^{4}$ Professor/a do Instituto de Dermatologia Professor Rubem David Azulay. Hospital Geral da Santa Casa de Misericórdia do Rio de \\ Janeiro, Brasil
}

RESUMO - A hanseníase históide representa uma forma atípica de apresentação da hanseníase virchowiana, descrita por Wade em 1963, com características clínicas e anatomopatológicas próprias, que no passado era considerada um marcador de resistência medicamentosa e recidiva. Clinicamente caracteriza-se por placas ou nodulos de aspecto queloidiforme. À microscopia óptica nota-se infiltrado predominantemente constituído por células fusiformes que por vezes simula neoplasia fibrohistiocítica, associada a poucos macrófagos espumosos e numerosos bacilos álcool-ácido-resistentes.

Relata-se um caso de hanseníase virchowiana com lesões clinicamente compatíveis com a forma históide, a fim de demonstrar e reforçar o seu grande polimorfismo clínico e a importância do seu conhecimento para diagnóstico e tratamento precoce, para interromper a cadeia de transmissão e diminuir a ocorrência de incapacidades irreversíveis causadas pela doença.

PALAVRAS-CHAVE - Hanseníase Virchowiana; Pele.

\section{Lepromatous Leprosy: Report of a Histoid-Like Case}

ABSTRACT - Histoid leprosy, described by Wade in 1963, represents an atypical variant of lepromatous leprosy, with its own clinical and pathological features. In the past, it was considered a marker of drug resistance and relapse. It presents as keloid-like plaque or nodular lesions. Optical microscopy shows few foamy macrophages and numerous alcohol-acid-resistant bacilli and an infiltrate composed predominantly of spindle cells, which can mimic fibrohistiocytic neoplasms.

We report a case of lepromatous leprosy with lesions clinically compatible with the histoid variant in order to demonstrate and reinforce the clinical polymorphism of this type of Hansen's disease and raise awareness for the need of an early diagnosis and treatment, therefore with the interruption of the transmission chain and reduction of the occurrence of irreversible leprosy-related disability.

KEYWORDS - Leprosy, Lepromatous; Skin.

\section{INTRODUÇÃO}

A hanseníase é uma doença infectocontagiosa crónica causada pelo Mycobacterium leprae.' Afeta inicialmente o sistema nervoso periférico devido ao neurotropismo do agente e posteriormente a pele, o que pode levar as sequelas e incapacidades funcionais conhecidas. ${ }^{2}$ Apresenta um amplo espectro clínico, que varia do pólo tuberculóide ao virchowiano, sendo muitas vezes considerada um grande desafio diagnóstico. ${ }^{2}$ Em 1963, Wade descreveu uma variante atípica de hanseníase virchowiana, a forma históide, com características clínicas e anatomopatológicas próprias. $^{3}$ Este artigo tem como objetivo relatar um caso clinicamente compatível com a hanseníase históide, cujo exame anatomopatológico demonstrou infiltrado inflamatório constituído apenas por macrófagos espumosos, porém sem o achado classicamente descrito de predomínio de células fusiformes, portanto uma forma de hanseníase virchowiana históide símile.
Correspondência: Felipe Tavares Rodrigues

Rua Visconde de Abaeté, 51 - Vila Isabel

Rio de Janeiro - RJ - Brazil.

Email: medftr@yahoo.com.br

DOI: https://dx.doi.org/10.29021/spdv.77.1.962
Recebido/Received

21 Junho/June 2018

Aceite/Accepted

25 Dezembro/December 2018 


\section{Caso Clínico}

\section{CASO CLÍNICO}

Paciente, género feminino, 48 anos, melanodérmica, natural do Rio de Janeiro, hipertensa e diabética em tratamento com metformina de 500 mg duas vezes por dia, que recorreu ao serviço de Dermatologia por "caroços pelo corpo" desde há 1 ano, assintomáticos mas com disseminação e aumento progressivos. Negava casos semelhantes na família e sinais e sintomas sistémicos associados.

Ao exame dermatológico apresentava pápulas e nódulos, amarelo-acastanhados, de superfície lisa e brilhante, bem delimitados, de tamanho variando entre 0,1-1 cm, consistência firme e aspecto queloidiforme, alguns de superfície ulcerada e/ou crostosa, localizados nos membros inferiores, membros superiores, parede abdominal, dorso, colo e face (Fig.s 1 e $2)$, mas sem madarose e/ou fácies leonina. $O$ teste de sensibilidade térmica, dolorosa e tátil nas lesões demonstrou hipoestesia térmica, descrita como a primeira sensibilidade a ser perdida na doença de Hansen por ser transmitida através de

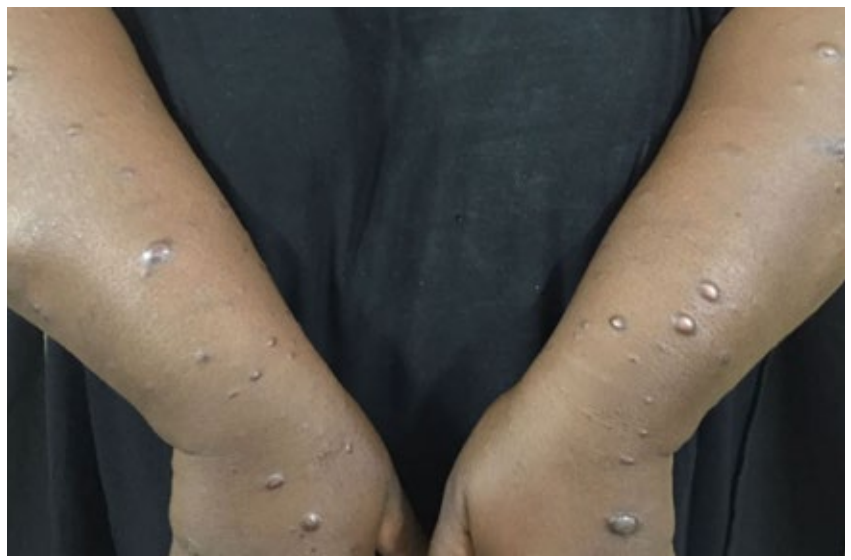

Figura 1 - Pápulas e nódulos, bem delimitados, de superfície lisa e brilhante, nos membros superiores.

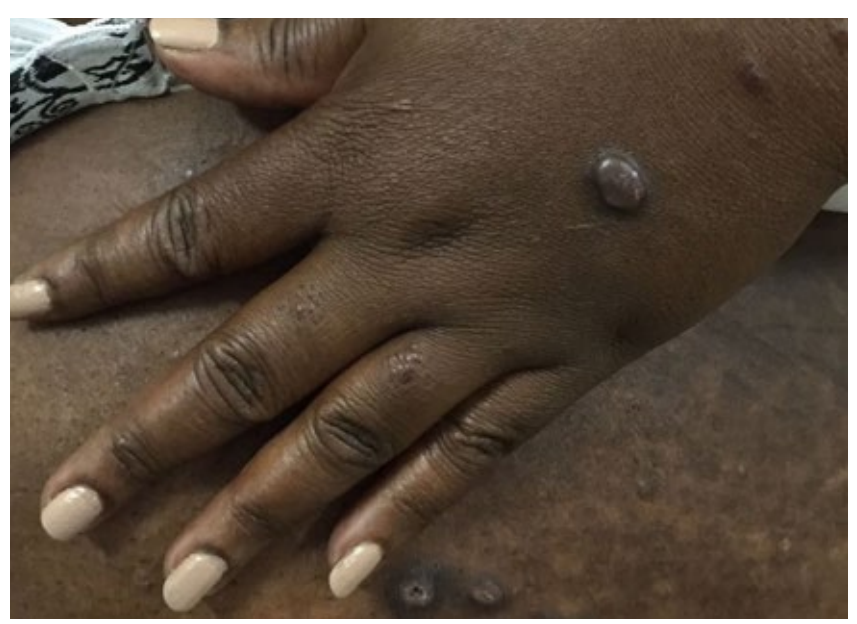

Figura 2 - Nódulo de aspecto queloidiforme no dorso da mão esquerda, alguns ulcerado ou com superfície crostosa. feixes nervosos finos. ${ }^{4}$ Diante da suspeição clínica de hanseníase multibacilar subtipo históide, foi realizada uma biópsia cutânea por punch de $0,5 \mathrm{~cm}$ de diâmetro numa das lesões nodulares contemplando uma boa amostra de tecido subcutâneo e sua inervação, com o cuidado de evitar o risco de artefatos de esmagamento obtidos durante a colheita. $O$ exame evidenciou retificação epidérmica e infiltração dérmica difusa de histiócitos xantomatosos contendo inúmeros bacilos álcool-ácido-resistentes, pontualmente formando globias, vistos pela técnica de Wade, compatível com hanseníase virchowiana com células vacuolizadas. Índice logarítmico da biópsia de 2,5 (Fig.s 3 e 4). A baciloscopia em lâmina de vidro de esfregaço intradérmico de quatro locais (lóbulo auricular direito, lóbulo auricular esquerdo, cotovelo direito e lesão cutânea) evidenciou índice baciloscópico de $2+$ e presença de globias, o que representa em média 1 a 10 bacilos de Mycobacterium leprae em cada 10 campos estudados na microscopia. Pela avaliação neurológica simplificada não foram detectados dor e espessamento dos nervos periféricos. Entretanto, pelo teste sensitivo motor dos membros superiores e inferiores, através de um estesiómetro, observou-se uma neuropatia acometendo o ramo plantar medial do nervo tibial, com respectiva diminuição da sensibilidade tátil protetora nos pés, classificada como grau 1 de incapacidade.

Foi iniciada poliquimioterapia multibacilar (PQT-MB) com rifampcina $600 \mathrm{mg}$ dose mensal supervisionada, clofazimina $300 \mathrm{mg}$ dose mensal supervisionada mais 50 mg dose diária

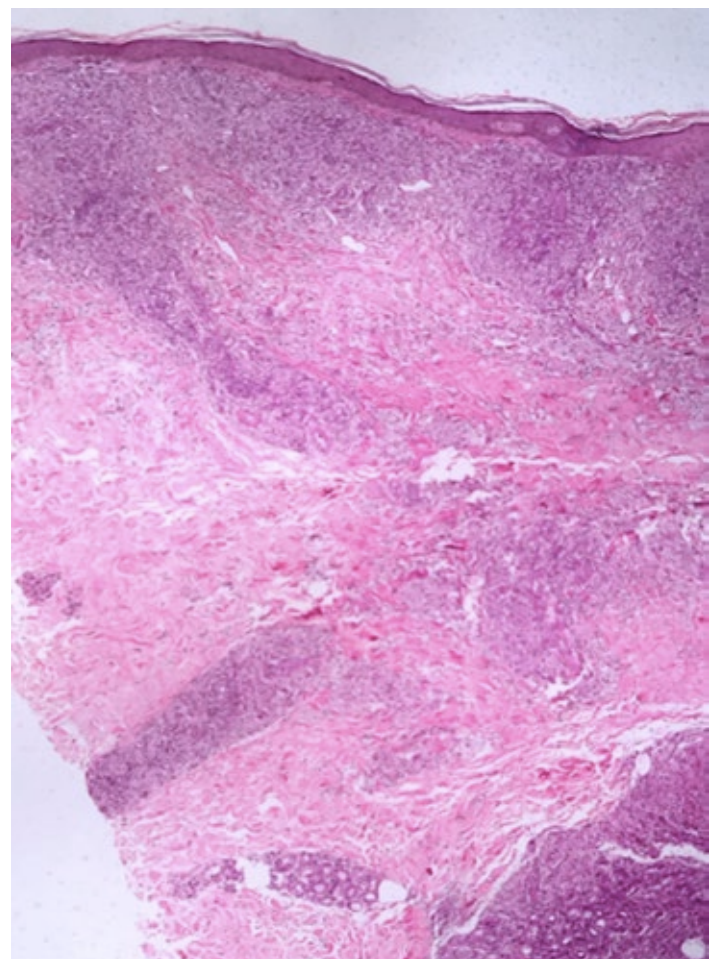

Figura 3 - Infiltrado inflamatório perivascular superficial e profundo, perianexial e confluente na derme alta, com formação de faixa de Unna. $(\mathrm{HE}, 40 \mathrm{X})$. 


\section{Caso Clínico}

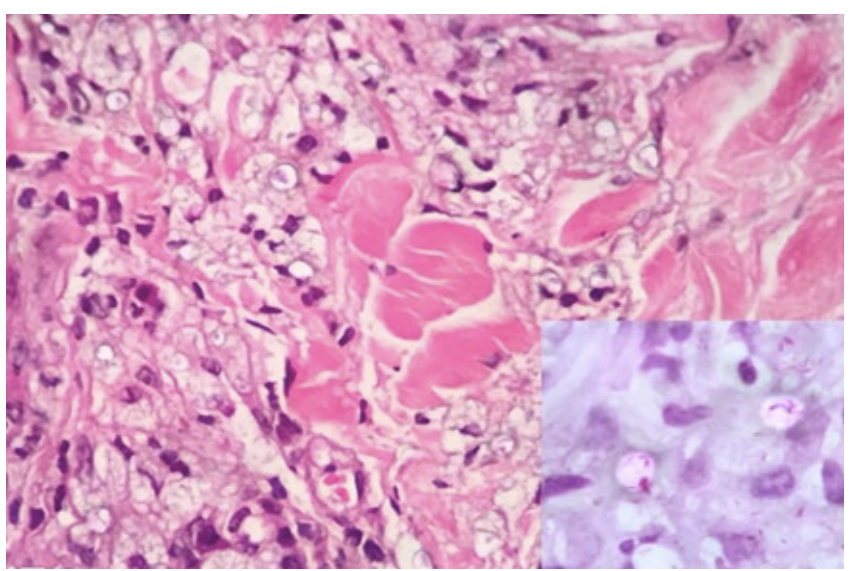

Figura 4 - Infiltrado inflamatório predominantemente constituído por macrófagos espumosos ( $\mathrm{HE}, 400 \mathrm{X})$. No detalhe observam-se numerosos bacilos álcool-ácido-resistentes, com a formação de globias (Wade, 1000X).

em casa e dapsona $100 \mathrm{mg}$ dose mensal supervisionada e diária em casa, com previsão de 12 doses. Houve uma melhoria importante das lesões nos primeiros seis meses de tratamento (Fig. 5) e regressão completa das mesmas no final do tratamento, com ausência de sequelas clínicas.

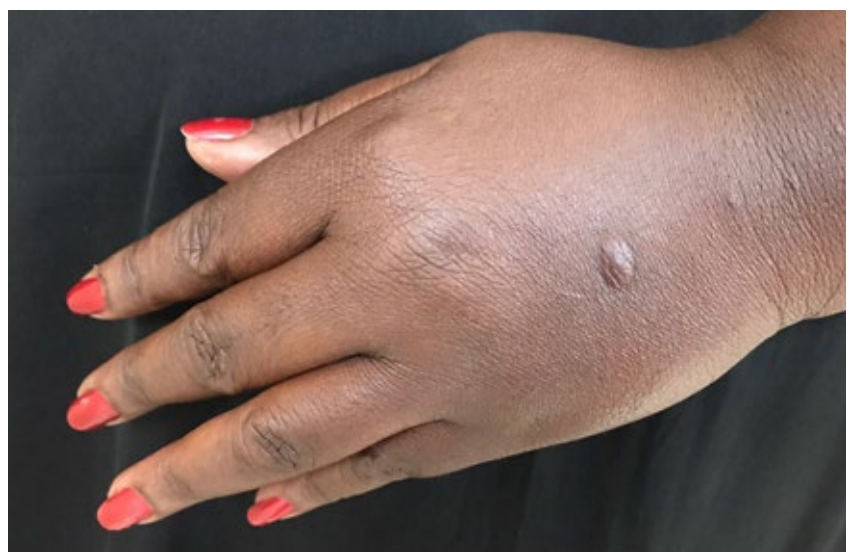

Figura 5 - Lesão cicatricial no dorso da mão esquerda após 6 meses de tratamento

\section{DISCUSSÃO}

A variante históide da hanseníase virchowiana é uma forma rara descrita inicialmente sobretudo em pacientes multibacilares em tratamento com monoterapia à base de sulfona em que os bacilos desenvolviam resistência medicamentosa à dapsona e recidivavam com essa forma. ${ }^{3}$ Entretanto, atualmente acredita-se que mesmo sendo rara em pacientes virgens de tratamento, esta forma de Hanseníase pode ocorrer "de novo", já não sendo considerada um marcador de recidiva. ${ }^{5}$ Além disso esta forma já não é atribuída à resistência medicamentosa, sendo responsiva à PQT-MB, como demonstrado por vários relatos na literatura. ${ }^{6,7}$ Acredita-se ainda que a sua etiopatogenia está associada a um decréscimo da imunidade celular sistémica com redução dos níveis de IL-2 e INF-y associado a um aumento local da imunidade celular e humoral, rica em macrófagos CD36+, basófilos e linfócitos TCD4+, contudo pobre em células dendríticas. Estas não apresentariam os antígenos adequadamente às células efetoras do sistema imune o que poderia explicar a origem das lesões hiperplásicas com um grande número de bacilos e pouca formação de globias. ${ }^{8-11}$ A diabetes mellitus tipo 2, um fator inflamatório e imunossupressor sistêmico conhecido, pode ter contribuído para a fisiopatologia da hanseníase virchowiana históide neste caso. ${ }^{12}$ Não há substrato científico que demonstre que a forma históide responde pior à terapêutica quando comparada às demais formas da hanseníase, sendo a PQT-MB o tratamento de primeira linha preconizado pela Organização Mundial de Saúde (OMS) para todas as formas multibacilares da doença, incluindo a forma históide, ${ }^{12}$ sendo bons os resultados como no presente caso.

A hanseníase multibacilar pode apresentar-se nas formas virchowiana clássica e dimorfa segundo os preceitos de Ridley e Jopling e, mais raramente, na forma virchowiana históide, como neste caso. A hanseníase dimorfa apresenta-se principalmente com placas eritematosas com aspecto violáceo ou ferruginoso formando típicas lesões foveolares ou manchas hipocrómicas ou eritematosas com bordas ferruginosas, com limite interno nítido e limites externos imprecisos. Já a hanseníase virchowiana clássica manifesta-se clinicamente por infiltração cutânea difusa com alopécia dos cílios e supercílios (madarose), além de lesões papulonodulares ou em placas, infiltradas, eritemato-acastanhadas, múltiplas, simétricas, de limites imprecisos, denominadas hansenomas/lepromas. Os locais de predileção são a face e a região auricular, levando a um facies característica denominada "facies leonina". 1,14

Tais características não foram encontradas na paciente descrita, sendo o quadro clínico compatível com a forma históide. Esta é caracterizada por lesões nodulares ou em placas bem delimitadas, assintomáticas, de superfície lisa brilhante e consistência firme, normocrómicas a amarelo-acastanhadas, de aspecto queloidiforme, que variam em tamanho e número, e localizam-se geralmente nos braços, dorso das mãos, nádegas, coxas, dorso e sobre as projeções ósseas, especialmente nos cotovelos e joelhos. ${ }^{8}$ As regiões palmo-plantares são frequentemente poupadas. ${ }^{9}$ Muitas vezes esta forma rara é subdiagnosticada, e erradamente as lesões são interpretadas como dermatofibroma, dermatofibrossarcoma protuberans, xantoma e até neurofibromas. ${ }^{15}$

Os achados histopatológicos da forma históide são típicos e assemelham-se aos de um tumor fibrohistiocítico, 8 caracterizando-se por atrofia epidérmica e área circunscrita onde se acumulam histiócitos fusiformes dispostos em padrão estoriforme, ricos em bacilos com características peculiares descritas como "habitus históides", porém sem a formação de globias. ${ }^{3}$ A histopatologia tem um papel fundamental na confirmação do diagnóstico e na conclusão do subtipo histológico da hanseníase históide: forma fusocelular pura, fusocelular com componente epitelióide e fusocelular com células vacuolizadas. Já na histopatologia da hanseníase virchowiana 


\section{Caso Clínico}

observa-se epiderme atrófica, uma fina camada de colagénio subepidérmico onde não há células de Virchow (faixa de Unna), um infiltrado dérmico perivascular, perianexial e perineural, monomórfico, constituído por histiócitos espumosos, denominados células de Virchow. No citoplasma dessas células encontram-se bacilos íntegros, aglomerados em globias., ${ }^{1,2}$

Apesar da paciente relatada se apresentar clinicamente com lesões tipo queloide características da forma históide, o exame histopatológico não demonstrou os achados histopatológicos mais característicos desta forma da doença. A presença de atrofia epidérmica, faixa de Unna, histiócitos xantomatosos repletos de bacilos agrupados em globias são as características clássicas da hanseníase virchowiana. Trata-se de um caso raro de dissociação clínico-histopatológica na hanseníase, interpretado como um caso de hanseníase históide fusocelular vacuolizado com componentes histopatológicos atípicos ou mais provavelmente um caso de hanseníase virchowiana históide-like. ${ }^{16}$

A hanseníase multibacilar apresenta grande polimorfismo clínico e é uma grande mimetizadora de outras doenças, pois pode apresentar-se com uma vasta variedade de lesões, - que pode dificultar e atrasar o diagnóstico, contribuindo para a manutenção da cadeia de transmissão e a ocorrência de complicações. Neste sentido, é importante estar atento às manifestações clínicas variadas da doença, para um diagnóstico e tratamento precoces.

Conflitos de interesse: Os autores declaram não possuir conflitos de interesse.

Suporte financeiro: $O$ presente trabalho não foi suportado por nenhum subsídio ou bolsa.

Confidencialidade dos dados: Os autores declaram ter seguido os protocolos do seu centro de trabalho acerca da publicação dos dados de doentes.

Direito a privacidade e consentimento escrito: Os autores declaram que pediram consentimento para usar as imagens no artigo.

Conflicts of interest: The authors have no conflicts of interest to declare.

Financing Support: This work has not received any contribution, grant or scholarship.

Confidentiality of data: The authors declare that they have followed the protocols of their work center on the publication of data from patients.

Privacy policy and informed consent: The authors declare that have the written informed consent for the use of patient's photos in this article.

Proveniência e revisão por pares: Não comissionado; revisão externa por pares

Provenance and peer review: Not commissioned; externally peer reviewed

\section{REFERÊNCIAS}

1. Fischer M. Leprosy - an overview of clinical features, diagnosis, and treatment. J Dtsch Dermatol Ges. 2017; 15:801-27. doi: 10.1111/ddg.13301.

2. Araújo MG. Leprosy in Brazil. Rev Soc Bras Med Tropical. 2003; 36:373-82.

3. Wade HW. The histoid variety of lepromatous leprosy. IntJ Leprosy. 1963; 31:129-42.

4. Brasil. Ministério da Saúde. Secretaria de Vigilância em Saúde. Departamento de Vigilância das Doenças Transmissíveis. Guia prático sobre a hanseníase [recurso eletrônico] / Ministério da Saúde, Secretaria de Vigilância em Saúde, Departamento de Vigilância das Doenças Transmissíveis. - Brasília: Ministério da Saúde, 2017. 68 p.

5. Desikan, KV, lyer CG. Histoid variety of lepromatous leprosy: a histopathologic study. Int J Lepr. 1972; 40:149-56.

6. Bhutani LK, Bedi TR, Malhotra YK, Kandhari KC, Deo MG.. Histoid leprosy in North India. Int J Lepr Other Mycobact Dis. 1974; 42:174-81.

7. Price EW, Fitzherbert H. Histoid variety of lepromatous leprosy. Int J Lepr. 1966; 34:367-74.

8. Kontochristopoulos GL, Aroni K, Panteleos DN, Tosca AD. Immunohistochemistry in histoid leprosy. Int J Dermatol. 1995;34:777-81.

9. Cuevas-Santos J, Contreras F, McNutt NS. Multibacillary leprosy: lesions with macrophages positive for $\mathrm{S} 100$ protein and dendritic cells positive for Factor 13a. J Cutan Pathol. 1998; 25:530-7.

10. Sehgal V, Srivastava G. Status of histoid leprosy - a clinical, bacteriological, histopathological and immunological appraisal. J Dermatol. 1987; 14:38-42.

11. Gupta SK. Histoid leprosy: review of the literature. Int J Dermatol. 2015;54:1283-8. doi: 10.1111/iid.12799.

12. Donath MY, Shoelson SE. Type 2 Diabetes as an inflammatory disease. Nat Rev Immun. 2011; 11:98-107. doi: 10.1038/nri2925.

13. Gross AM. Hanseníase no Brasil. Rev Soc Bras Med Trop. 2003; 36:373-82.

14. Sehgal VN, Srivastava G. Histoid leprosy. Int J Dermatol. 1985; 24:286-92.

15. Baslas RG, Gupta M, Arora SK, Mukhija RD, Misra RK. Palmar involvement in histoid leprosy. Indian J Lepr. 1992;64:193-5.

16. Manoharan R, Madhu R, Srinivasan MS. Histoid Hansen - A case report. J Indian Soc Tele Dermatol. 2008;2:12-6. 